\title{
Aspectos cognitivos e nível de atividade física de idosos
}

\author{
Camila da Cruz Ramos de Araujo1', Camila da Silveira², Joseani Paulini Neves Simas³, \\ Alessandra Zappelini ${ }^{4}$, Silvia Rosane Parcias ${ }^{5}$, Adriana Coutinho de Azevedo Guimarães ${ }^{6}$.
}

\begin{abstract}
RESUMO
Objetivo: Investigar os aspectos cognitivos e o nível de atividade física de idosos. Método: Estudo de corte transversal realizado com 125 idosos participantes de intervenções motoras e cognitivas no Centro de Atenção a Terceira Idade - São José/SC. Para este estudo foram aplicados os seguintes instrumentos: a)Informações gerais; b)Situação socioeconômica (ABEP); c)Percepção de saúde; d)Questionário Internacional de Atividade Física; e)Escala de depressão geriátrica; f)Mini-exame do estado mental (MEEM); g)Teste de traços; h)Teste do relógio. Resultados: $70 \%$ dos idosos foram classificados como suficientemente ativos (ativo/muito ativo). No Teste de Trilhas A $(85,1 \%)$ e B $(81,6 \%)$ e no teste do relógio (76\%) verificou-se baixo desempenho, caracterizando déficit cognitivo. No MEEM 53,6\% apresentaram comprometimento (leve/moderado/grave) e 46,4\% sem comprometimento, sendo o comprometimento leve o mais frequente (45,6\%), com destaque para os idosos na faixa etária dos $70-79$ anos ( $p=0,047)$. Em relação a depressão $74,4 \%$ não apresentaram suspeita de depressão. Conclusão: A maioria dos idosos não apresentam indicativos de depressão, sendo suficientemente ativos, com baixo desempenho cognitivo (déficit de atenção e funções executivas) e comprometimento do estado mental.
\end{abstract}

Descritores: Cognição; Atividade Física; Idoso.

\section{Cognitive aspects and physical activity level among older adults}

\begin{abstract}
Objective: To investigate the cognitive and physical activity for older adults. Method: Cross-sectional study conducted with 125 elderly participants of motor and cognitive interventions in the Center for the Elderly - São José / SC. For this study the following instruments were applied: a) General Information b) Socioeconomic Status (ABEP); c) Perceived health; d) International Physical Activity Questionnaire; e) Geriatric Depression Scale; f) Mini-mental State Examination; g) Test traits; h) Test the clock. Results: $70 \%$ of subjects were classified as sufficiently active (active / very active). In the Trail Making Test A $(85,1 \%)$ and B $(81,6 \%)$ and the clock drawing test $(76 \%)$ showed low performance, featuring cognitive impairment. MMSE $53.6 \%$ had impaired (mild / moderate / severe) and $46.4 \%$ without commitment, being mild impairment the most frequent $(45.6 \%)$, especially for the elderly in the age group of $70-79$ years $(p=0.047)$. In relation to $74.4 \%$ depression showed no suspicion of depression. Conclusion: Most elderly do not present indicative of depression, being sufficiently active, with low cognitive performance (attention deficit and executive functions), and impaired mental state
\end{abstract}

Descriptors: Cognition; Physical Activity; Elderly.

\footnotetext{
${ }^{1}$ Mestranda em Ciências do Movimento Humano na Universidade do Estado de Santa Catarina (UDESC), Florianópolis, SC, Brasil.

${ }^{2}$ Graduada em Educação Física pela Universidade do Estado de Santa Catarina (UDESC), Florianópolis, SC, Brasil.

${ }^{3}$ Mestre em Educação Fisica pela Universidade Federal de Santa Catarina (UFSC), Florianópolis, SC, Brasil.

${ }^{4}$ Graduada em Psicologia pela Universidade Federal de Santa Catarina (UFSC), Florianópolis, SC, Brasil.

${ }^{5}$ Doutora em Neurociências pela Universidad de Zaragoza (UNIZAR), Zaragoza, Espanha.

${ }^{6}$ Doutora em Motricidade Humana - Área da Saúde e Condição Física pela Universidade Técnica de Lisboa (UTL), Lisboa, Portugal.
} 


\section{Introdução}

O processo de envelhecimento acarreta mudanças fisiológicas, nomeadamente, alterações sensoriais, déficits cognitivos, diminuição dos reflexos e dos níveis de concentração. Ainda, o indivíduo idoso reage mais lentamente a estímulos, refletindo em alterações na memória, criatividade, atenção e iniciativa ${ }^{1,2}$.

Além disso, alterações físicas, como à diminuição das capacidades físicas e aptidões funcionais do organismo também são observadas durante esse processo ${ }^{3}$.

O desempenho e o funcionamento cognitivo dos idosos têm recebido cuidado dos profissionais da área da saúde ${ }^{2,4,5}$. Essa atenção se justifica pelo fato de que alterações nas funções cognitivas podem comprometer o bem-estar biopsicossocial do idoso $e$ o dia a dia de seus familiares ${ }^{4}$. A função cognitiva pode ser entendida como as fases do processo de informação, como percepção, aprendizagem, memória, atenção, vigilância, raciocínio e solução de problemas, além do funcionamento psicomotor ${ }^{6}$.

Com o envelhecimento as perdas cognitivas, de maneira geral, se iniciam por prejuízos nos processos de memória, que a longo prazo refere-se à quantidade e à qualidade da informação que uma pessoa é capaz de reter sobre si mesma ao longo do tempo ${ }^{5,7}$. O declínio da memória pode variar de pequenos esquecimentos, os quais não geram grandes consequências para a manutenção da vida social e para a realização das atividades de maneira independente, a casos mais severos, como as demências, em que há um comprometimento importante das funções congnitivas ${ }^{8}$.

Por meio da manutenção de um estado de saúde saudável, as capacidades motoras são fundamentais na realização da atividade física, influenciando na execução de tarefas diárias, afastando os fatores de risco comuns à terceira idade e promovendo uma melhor qualidade de vida a estes indivíduos ${ }^{9}$. Além das tarefas diárias, a atividade física que é associada com a melhora ou manutenção do bem-estar físico e redução do risco de doenças cardiovasculares, também pode representar uma importante contribuição não farmacológica no sentido de atenuar a taxa de declínio cognitivo e motor em relação ao envelhecimento ${ }^{10}$.

Apesar dos benefícios, ainda é alarmante os patamares que a prevalência de inatividade física tem alcançado no mundo. Um estudo verificou que $31 \%$ dos adultos no mundo não praticam a quantidade mínima de atividade física recomendada pela OMS ${ }^{11}$. Com o avanço da idade, aumenta-se a inatividade física. Um estudo verificou que $47 \%$ dos idosos do mundo não praticam atividade física suficiente, tornando-a a quarta principal causa de morte no mundo ${ }^{12}$.

Como reflexo dessa inatividade física e da influência que a mesma tem nos aspectos cognitivos, há um crescimento de programas e locais que propiciam experiências cognitivas enriquecedoras como forma de ocupação do tempo livre dos idosos, promovendo atividades estimulantes, que visam à promoção da sociabilidade, colabora para a autonomia, propiciam amizades, além de promover um aumento da autoestima, resgatar valores pessoais, favorecer a adoção de um estilo de vida mais ativo, pois nestes são realizadas atividades físicas, cognitivas, lazer, culturais, manuais, artísticas ${ }^{13,14}$. Para alguns idosos a participação em centros de convivências pode estar relacionada à diminuição do estresse, aumento dos sentimentos e emoções positivas, controle da saúde, ampliação das ofertas de suporte social, exercício da habilidade de resolução de problemas e manutenção das capacidades cognitivas e motoras ${ }^{14,15}$.

Torna-se extremamente importante monitorar e rastrear a progressão dos sintomas de déficit cognitivo e conhecer as demandas e potencialidades de saúde e de vida dos idosos, a fim de planejar possíveis intervenções, contribuindo na elaboração de políticas públicas de atenção a saúde para essa população ${ }^{5}$. Ademais, 0 desuso das habilidades físicas e mentais frequentemente associadas com o envelhecimento tem resultados sociais e econômicos que afetam tanto na manutenção da capacidade funcional, como na independência do idoso. Isto conduz ao propósito de considerar o exercício físico como uma relevante estratégia para melhorar o funcionamento motor e cognitivo na velhice ${ }^{16}$.

Nesse sentido e de acordo com o exposto acima, pretende-se investigar os aspectos cognitivos e o nível de atividade física de idosos participantes de intervenções motoras e cognitivas.

\section{Metodologia e avaliação}

O estudo de corte transversal não probabilístico por acessibilidade foi realizado no município de São José em Santa Catarina de 2011 a 2013, № 38/2011 do Comitê de Ética em Pesquisa da Universidade do Estado de Santa Catarina. Todos os participantes assinaram o termo de consentimento livre e esclarecido. A amostra por acessibilidade foi composta de 125 idosos matriculados no Centro de Atenção à Terceira Idade, com média de idade de 70,3 anos. 
Esses idosos participaram de intervenções motoras e cognitivas duas vezes na semana com aproximadamente 1 hora de duração, durante seis meses. As intervenções e avaliações foram mediadas por uma equipe composta pela: Professora responsável da UDESC, a psicóloga do Centro de Atenção a Terceira Idade - São José/SC e estudantes de educação física e fisioterapia.

Anteriormente da realização das intervenções, foi aplicado em forma de entrevista, um questionário com oito instrumentos previamente validados para avaliação dos sujeitos:

1) Identificação pessoal: sete perguntas para identificar sexo, idade, escolaridade, estado civil, aposentadoria e moradia;

2) Situação socioeconômica: por meio do instrumento adotado pela Associação Brasileira de Estudos Populacionais - Critério de Classificação Econômica Brasil ${ }^{17}$ que é considerado o principal instrumento de segmentação da população segundo seu poder de compra, o qual classifica a população em classes econômicas A, B, C, D e E, por meio da pontuação obtida e renda mensal);

3) Percepção de Saúde Geral - segundo modelo da questão contida no BRFSS - Behavioral Risk Factors Surveillance System Questionaire ${ }^{18}$;

4) Nível de Atividade Física - Questionário Internacional de Atividade Física (International Physical Activity Questionnaire) IPAQ versão curta. O IPAQ é um instrumento auto-administrável, composto por seis itens, que procura verificar o número de vezes em que o sujeito praticou pelo menos 10 minutos contínuos de caminhada, atividade física moderada e vigorosa, na última semana, em diversos envolvimentos, nomeadamente, laboral, doméstico, lazer, recreativo e desportivo. A análise e classificação dos resultados segue as orientações do Centro de Estudos do Laboratório de Aptidão Física de São Caetano do Sul - CELAFISCS, que é o Centro Coordenador do IPAQ no Brasil ${ }^{19}$. De acordo com a quantidade de atividade física realizada, os indivíduos podem ser classificados como sedentários, insuficientemente ativos, ativos, ou muito ativos;

5) Escala de Depressão Geriátrica (GDS-30). Amplamente utilizada e validada como instrumento diagnóstico de depressão em pacientes idosos. É um teste para detecção de sintomas depressivos no idoso, com 30 perguntas negativas/afirmativas onde o resultado de 10 ou mais pontos diagnostica depressão ${ }^{20}$.

6) Mini-Exame do Estado Mental (MEEM): traduzido e adaptado para a língua portuguesa ${ }^{21}$, largamente utilizado na pesquisa e na clínica em indivíduos idosos obtendo um índice de confiabilidade de 0,85. 0 objetivo deste instrumento é avaliar funções cognitivas específicas como: orientação temporal, orientação espacial, memória imediata, atenção, cálculo, evocação e linguagem. $O$ escore do MEEM pode variar de um mínimo de 0 pontos, o qual indica o maior grau de comprometimento cognitivo dos indivíduos, até um total máximo de 30 pontos, o qual, por sua vez, corresponde a melhor capacidade cognitiva.

7) Teste de Trilhas ou Trail Making Test: Avalia a atenção, sequenciamento, flexibilidade mental, busca visual e função motora. É composto de duas partes (Trilhas A e Trilhas B), e sua realização é precedida por um treinamento curto do percurso a ser executado. Na forma $\mathrm{A}$ o treino consiste em 8 círculos com números cardinais. 0 teste em si possui 25 círculos, numerados de 1 a 25, distribuídos ao acaso, que devem ser unidos em uma linha contínua, ou seja, sem 0 examinado tirar o lápis do papel. Na forma $B 13$ números e 12 letras, devem ser unidos alternadamente (1-A; 2-B...). A forma $B$ também possui um treino que tem início com 1-A e segue até 4-B, ou seja, é pedido ao examinado que faça os pares número e letra, seguindo a sequência. É relevante que ambos os testes tenham as indicações de início e fim nos círculos correspondentes. 0 teste é encerrado após 3 erros ou cinco minutos ${ }^{22}$. Para esse estudo, os valores de referência utilizados, por faixa etária, seguiram os dados normativos de Tombaug ${ }^{23}$. Indivíduos com tempo acima do valor de referência são classificados como abaixo do padrão (déficit de atenção) e os abaixo do valor de referência são classificados como acima do padrão (nível de atenção normal).

8) Teste do Relógio: avalia a função cognitiva e disfunções executivas, que podem preceder os distúrbios de memória nas demências. $O$ desenho do relógio (clock drawing) consiste em um círculo pré-desenhado no qual o examinando deve colocar os números e os ponteiros indicando $11 \mathrm{~h} 10 \mathrm{~min}$. O modo de correção desse teste utiliza um detalhado sistema qualitativo e quantitativo dos erros cometidos pelo examinando. Esse sistema de escore classifica os erros em sete categorias amplas: 
omissão, ausência, distorção, substituição, adição e rotação. Para fazer a classificação e a pontuação, são analisados erros em relação ao desenho da face do relógio, dos números, dos ponteiros e dos espaços entre os números. Atribui-se um ponto para cada erro e o escore máximo é ilimitado, embora raramente sejam vistos valores superiores a 31 pontos ${ }^{24}$.

Para análise dos dados, utilizou-se o programa SPSS 20.0 mediante estatística descritiva (frequência relativa e absoluta, média e desvio padrão) para sintetizar os valores, permitindo dessa forma que se tenha uma visão global da variação dos valores obtidos. Para avaliação dos testes cognitivos entre as três faixas etárias dos idosos participantes, utilizou-se o teste do Qui quadrado.

\section{Resultados}

Observa-se na Tabela 1, que $88 \%$ dos participantes são mulheres, aposentados (79,\%), casados (36\%), e que frequentaram a escola até o primário (atual $1^{\circ}$ ao $5^{\circ}$ ano) (41\%). Em relação ao nível socioeconômico, a maioria está entre o nível baixo e médio, representadas pelos estratos C e B, com $47 \%$ e $33 \%$ respectivamente.

Tabela 1 - Caracterização dos idosos participantes do estudo, São José - SC. 2013

\begin{tabular}{|c|c|c|}
\hline Variáveis & $f$ & $\%$ \\
\hline \multicolumn{3}{|l|}{ Sexo $(n=125)$} \\
\hline Masculino & 15 & 12,0 \\
\hline Feminino & 110 & 88,0 \\
\hline \multicolumn{3}{|l|}{ Escolaridade $(n=125)$} \\
\hline Nenhuma & 6 & 4,8 \\
\hline Primário & 51 & 40,8 \\
\hline Ginásio ou $1^{\circ} \mathrm{Grau}$ & 27 & 21,6 \\
\hline $2^{\circ} \mathrm{Grau}$ & 25 & 20,0 \\
\hline Superior & 16 & 12,8 \\
\hline \multicolumn{3}{|l|}{ Estado Conjugal ( $n=125)$} \\
\hline Casado/Morando junto & 45 & 36,0 \\
\hline Viúvo & 42 & 33,6 \\
\hline Divorciado / Separado & 26 & 20,8 \\
\hline Solteiro & 12 & 9,6 \\
\hline \multicolumn{3}{|l|}{ Aposentadoria $(n=125)$} \\
\hline Sim & 99 & 79,2 \\
\hline Não & 26 & 20,8 \\
\hline \multicolumn{3}{|l|}{ Nível Socioeconômico (n=125) } \\
\hline A & 7 & 5,6 \\
\hline B & 41 & 32,8 \\
\hline C & 59 & 47,2 \\
\hline$D$ & 18 & 14,4 \\
\hline
\end{tabular}

f: frequência; Estatística descritiva; Frequência relativa e absoluta

A percepção da saúde é relatada na tabela 2, na qual é possível verificar que os participantes do estudo consideraram sua saúde boa (39\%). Aproximadamente $90 \%$ dos idosos apresentaram problemas de saúde e $70 \%$ foram considerados suficientemente ativos (ativo + muito ativo). 
Tabela 2 - Percepção do estado de saúde e nível de atividade física dos idosos participantes do estudo, São José - SC. 2013

\begin{tabular}{c|c|c}
\hline Variáveis & f & $\%$ \\
\hline Percepção de saúde (n=125) & 10 & 8,0 \\
\hline Excelente & 16 & 12,8 \\
\hline Muito Boa & 49 & 39,2 \\
\hline Boa & 42 & 33,6 \\
\hline Regular & 8 & 6,4 \\
\hline Ruim & 112 & 89,6 \\
\hline Problemas de Saúde (n=125) & 13 & 10,4 \\
\hline Sim & & 30,4 \\
\hline Não & 38 & 31,4 \\
\hline Nível de Atividade Física (n=125) & 39 & 38,4 \\
\hline Insuficientemente ativo & 48 & \\
\hline Ativo & & \\
\hline Muito ativo & & \\
\hline
\end{tabular}

f: frequência; Estatística descritiva; Frequência relativa e absoluta

Em relação a tabela 3, mais da metade dos participantes apresentaram algum comprometimento do estado mental (53,6\%), sendo o comprometimento leve o mais frequente $(45,6 \%)$, com destaque para os idosos na faixa etária dos $70-79$ anos ( $p=0,047)$. Apesar de não apresentarem diferença significativa, os idosos mais jovens (60-69 anos) apresentaram déficit de atenção nas duas partes do teste de trilhas, com respectivamente $87,1 \%$ para parte $A$ e $82,3 \%$ para parte $B$. Para o teste do relógio essa mesma faixa etária classificou-se com anormalidade $(45,2 \%)$ e não apresentou suspeita de depressão $(75,8 \%)$.

Tabela 3 - Avaliação da cognição dos idosos participantes do estudo São José - SC. 2013

\begin{tabular}{|c|c|c|c|c|c|}
\hline Variáveis & Total $n(\%)$ & $69-69$ anos $n(\%)$ & $70-79$ anos $n(\%)$ & $80-89$ anos $n(\%)$ & Valor $p$ \\
\hline \multicolumn{6}{|l|}{ Estado Mental $(n=125)$} \\
\hline Comprometimento grave & $1(0,8)$ & - & - & $1(7,1)$ & \multirow{4}{*}{$0,047^{*}$} \\
\hline Comprometimento moderado & $9(7,2)$ & $2(3,2)$ & $6(12,2)$ & $1(7,1)$ & \\
\hline Comprometimento leve & $57(45,6)$ & $25(40,3)$ & $26(53,1)$ & $6(42,9)$ & \\
\hline Sem comprometimento & $58(46,4)$ & $35(56,5)$ & $17(34,7)$ & $6(42,9)$ & \\
\hline \multicolumn{6}{|l|}{ Teste de Traços A (n=125) } \\
\hline Nível de atenção normal & $18(14,4)$ & $8(12,9)$ & $8(16,3)$ & $2(14,3)$ & \multirow{2}{*}{$0,934^{*}$} \\
\hline Déficit de atenção & $107(85,6)$ & $54(87,1)$ & $41(83,7)$ & $12(85,6)$ & \\
\hline \multicolumn{6}{|l|}{ Teste de Traços B $(n=125)$} \\
\hline Nível de atenção normal & $23(18,4)$ & $11(17,7)$ & $7(14,3)$ & $5(35,7)$ & \multirow{2}{*}{0,213} \\
\hline Déficit de atenção & $102(81,6)$ & $51(82,3)$ & $42(85,7)$ & $9(64,3)$ & \\
\hline \multicolumn{6}{|l|}{ Teste do Relógio ( $n=125)$} \\
\hline Anormalidade & $59(47,2)$ & $28(45,2)$ & $25(51,0)$ & $6(42,9)$ & \multirow{3}{*}{$0,832^{*}$} \\
\hline Suspeita de anormalidade & $36(28,8)$ & $17(27,4)$ & $15(30,6)$ & $4(28,6)$ & \\
\hline Desempenho normal & $30(24,0)$ & $17(27,4)$ & $9(18,4)$ & $4(28,6)$ & \\
\hline \multicolumn{6}{|l|}{ Depressão (n=125) } \\
\hline Sem suspeita de depressão & $93(74,4)$ & $47(75,8)$ & $34(69,4)$ & $12(85,7)$ & \multirow{2}{*}{$0,491^{*}$} \\
\hline Com suspeita de depressão & $32(25,6)$ & $14(24,2)$ & $15(30,6)$ & $2(14,3)$ & \\
\hline
\end{tabular}

Qui quadrado; *Exato de Fisher 


\section{Discussão}

A prática regular de atividade física proporciona inúmeros benefícios relacionados à saúde ${ }^{25}$, podendo ainda exercer um efeito positivo sobre vários processos cognitivos em idosos ${ }^{26}$.

Diante desta perspectiva e de acordo com o principal objetivo do estudo que investigou os aspectos cognitivos e o nível de atividade física de idosos, os resultados indicam que a maioria dos participantes deste estudo $(53,6 \%)$ apresentaram algum comprometimento no estado mental, com destaque para o variável comprometimento leve (45,6\%). Dividindo pela faixa etária dos indivíduos, aqueles pertencentes a faixa etária de 70-99 anos apresentaram o maior percentual de comprometimento leve $(53,1 \%)$. Utilizando o mesmo instrumento de medida (MEEM), Machado et al. ${ }^{27}$ e Leite et al. ${ }^{28}$ também detectaram declínio cognitivo nos idosos, com uma prevalência de respectivamente $36,5 \%$ e $23,5 \%$. Neste sentido, o declínio cognitivo pode estar relacionado ao comprometimento da capacidade funcional destes indivíduos, acarretando perda de autonomia e independência, e consequente redução da qualidade de vida ${ }^{27}$. Esses achados não corroboraram com a literatura, pois a mesma relata que participação em centros de convivências pode estar relacionada à diminuição do estresse, aumento dos sentimentos e emoções positivas, controle da saúde, ampliação das ofertas de suporte social, exercício da habilidade de resolução de problemas e manutenção das capacidades cognitivas e motoras ${ }^{14,15}$.

Os idosos do nosso estudo apresentaram déficit abaixo do esperado nas duas partes do trail making test (A-B) com 85,6\% e $81,6 \%$ respectivamente, os valores de referencia por faixa etária utilizada seguiram os dados normativos de Tombaugh ${ }^{29}$. Isso pode estar relacionado ao fato de que a falta de atenção agrava com o envelhecimento ${ }^{30}$. Outro estudo também constatou piora no desempenho em testes de função executiva com o avançar da idade ${ }^{31}$. No entanto, nossos resultados indicaram que os idosos mais jovens (60-69 anos) apresentaram os maiores déficits no teste de trilhas, com $87,1 \%$ para parte Ae $82,3 \%$ para parte B. Dessa forma, nossos resultados diferem dos resultados encontrados no estudo realizado no município do norte do Rio Grande do Sul o qual identificou que os idosos de 80 anos ou mais de idade apresentaram o maior percentual de declínio $\operatorname{cognitivo}^{28}$. Ainda, Valle et al ${ }^{32}$ em seu estudo de base populacional sobre saúde de idosos, observaram que os escores mais baixos do MEEM foram apresentadas pelos idosos pertencentes a faixa etária mais velha (> 80 anos).

O nível de escolaridade é um fator de proteção ao envelhecimento que pode atenuar, nas habilidades cognitivas, os efeitos do declínio neural associados ao envelhecimento ${ }^{33}$. Nosso estudo apresentou um nível de escolaridade baixo (40,8\% com primário completo), podendo ser um dos fatores para o baixo desempenho cognitivo apresentado. Esse menor nível de escolaridade é comum na população idosa brasileira e isso pode afetar o desempenho cognitivo. Diniz ${ }^{34}$ comenta que a aquisição do conhecimento a respeito das estruturas fonológica e gramatical da língua, alcançada com o aprendizado da leitura e da escrita, ocasiona mudanças arquiteturais e funcionais no cérebro humano, e influencia não somente a habilidade linguística de um indivíduo, mas também na maneira de processar e representar informações, como a habilidade de processar informações espaciais, de nomear objetos em duas dimensões, a memória de trabalho, o pensamento lógico e abstrato.

Um estudo realizado pelo SABE - Saúde, Bem-Estar e Envelhecimento em 2.143 idosos residentes na cidade de São Paulo com média de idade de 69 anos, identificou que $60 \%$ destes idosos têm menos de sete anos de escolaridade e que os problemas cognitivos observados em $11 \%$ da amostra foram mais frequentes em idosos que nunca frequentaram escola (17\%), do que em idosos com mais de sete anos de estudos ${ }^{35}$. Leite et al. ${ }^{36}$ relataram que quanto menor o nível de escolaridade dos idosos, mais sintomas depressivos eles apresentam com o decorrer da idade.

Neste estudo não foi verificado indicativos de depressão na maioria dos idosos $(74,4 \%)$, destacando-se os idosos pertencentes a faixa etária de 60-69 anos (75,8\%). É importante ressaltar que a baixa incidência de sintomas depressivos dos idosos deste estudo pode estar relacionada com a participação ativa em um centro de convivência, o que permite uma maior integração social e adesão a prática de atividades físicas regulares. Carvalho et al. ${ }^{37}$ relatam que a falta de atividades diárias e locais adequados que incentivem e estimulem os idosos, aumenta os sentimentos de não utilidade, convívio com a solidão e a perda de sentido de vida, contribuindo para a incidência de sintomas depressivos.

O declínio cognitivo levado pelo envelhecimento também é prejudicado pelo nível socioeconômico do indivíduo, sendo este um fator crucial para garantir aos idosos um envelhecimento digno ${ }^{38}$. Idosos com baixa renda podem apresentar falta de estímulo cognitivo e incentivo para a prática de atividades física, uma vez que possuem menores possibilidades e falta de informações sobre os benefícios para a saúde ${ }^{39}$. Em decorrência a vida atual, os idosos estão sustentando suas casas com única fonte de renda, ou seja, o valor da aposentadoria que é hoje inferior aos vencimentos de seu período produtiv ${ }^{40}$.

A percepção do estado de saúde neste estudo caracterizou-se de maneira geral como positiva, o que é desejável, pois segundo Carvalho et al. ${ }^{37}$ é uma referência global para a qual a pessoa se considera, capaz de identificar aspectos da 
saúde física, cognitiva e emocional. Indivíduos com percepção negativa do estado de saúde têm maior risco de morte, em comparação com as que relatam uma saúde positiva. A percepção, portanto, vem sendo utilizada nas pesquisas por ser um forte indicador de mortalidade e declínio funcional e cognitivo ${ }^{37}$. Salientamos que os idosos deste estudo participam de grupos de convivência, que oferecem atividades física, atividades culturais, sociais, assistência médica, nutricional e psicológica que contribuem positivamente na saúde e percepção do idoso.

Os nossos resultados em relação ao nível de atividade física indicam que aproximadamente $69,8 \%$ dos idosos classificam-se como suficientemente ativos (ativos+muito ativos) e apenas 30\% como insuficientemente ativos, neste sentido, além dos inúmeros benefícios relacionados à saúde em geral ${ }^{41}$ a atividade física parece exercer um efeito positivo sobre vários processos cognitivos em idosos ${ }^{26}$. Especificamente a atividade física de caráter lúdico desempenha um papel importante no processo de aprendizagem cognitiva dos idosos, preparando-os para o autocuidado, a autonomia e a independência. Além disso, para os participantes, estas atividades podem parecer apenas brincadeiras, mas no processo de aprendizagem tem um propósito maior que é levar a uma percepção cognitiva, transformando o concreto em abstrato e proporcionando ainda, momentos de alegria e descontração ${ }^{33}$. Segundo Oliveira ${ }^{7}$ idosos engajados em atividades que desenvolvem as suas potencialidades cognitivas integradas às motoras, apresentam mudanças no campo cognitivo e boas condições de saúde.

\section{Considerações Finais}

Após investigar os aspectos cognitivos e o nível de atividade física dos idosos participantes deste estudo, observou-se que, apesar de exibirem problemas de saúde, a maioria dos participantes percebeu positivamente a sua saúde e não apresentou indicativos de depressão. Quanto ao nível de atividade física, os mesmos são suficientemente ativos, no entanto revelaram baixo desempenho cognitivo e algum comprometimento do estado mental.

Desta forma ao se traçar intervenções que visem à promoção e educação em saúde para idosos, sugere-se considerar a realização de atividades físicas e cognitivas nas mesmas intervenções, pois somente com ações construídas e executadas considerando o individuo no seu aspecto global e respeitando as suas individualidades, é que talvez sejam reinventados novos caminhos para promover um aumento na qualidade de vida e saúde dos idosos.

\section{Referências Bibliográficas}

1. Mazo GZ, et al. Tendência a estados depressivos em idosos praticantes de atividade física. Rev bras cineantropom desempenho hum. 2005; 7: 45-49.

2. Miranda LM de, Farias SF. A influência do exercício físico na atividade cerebral do idoso. Lect educ fís Deportes. 2008; 13: 01-10.

3. Von Bonsdorff MB, Rantanen T. Progression of functional limitations in relation to physical activity: a life course approach. Euro Rev Aging Phy Activity 2011; 8(1):23-30

4. Souza JN de, Chaves EC. O efeito do exercício de estimulação da memória em idosos saudáveis. Rev esc enferm USP. 2005; 39(1): 13-19.

5. Leite MT, et al . Estado cognitivo e condições de saúde de idosos que participam de grupos de convivência. Rev. Gaúcha Enferm. 2012; 33(4): 64-71.

6. Antunes HKM, Santos et al. Exercício físico e função cognitiva: uma revisão. Rev Bras Med Esporte. 2006; 12(2): 108-114.

7. Oliveira CS, et al. Oficina de Educação, Memória, Esquecimento e Jogos Lúdicos para a Terceira Idade. Revista Ciência em Extensão. 2012; 8(1): 8-17.

8. Charchat FH, et al. Declínio da capacidade cognitiva durante o envelhecimento. Rev Bras Psiquiatr. 2005; 27(12): $79-82$.

9. Katzer JI, Antes DL, Corazza ST. Coordenação Motora em Idosas. Conscientiae Saúde. 2012; 11(1): 159-163.

10. Hernandez SSS, et al. Efeitos de um programa de atividade física nas funções cognitivas, equilíbrio e risco de quedas em idosos com demência de Alzheimer. Rev Brás fisioter. 2010; 14(1): 68-74.

11. Hallal PC, et al. Global physical activity levels: surveillance progress, pitfalls, and prospects. Lancet. 2012; 380(9838): $247-257$

12. Kohl, HW, et al. The pandemic of physical inactivity: global action for public health. Lancet. 2012; 380(9838): 294-305.

13. Streit IA, et al. Aptidão física e ocorrência de quedas em idosos praticantes de exercícios físicos. Rev bras ativ fís saúde. 2011; 6(4): 346-352. 
14. Yassuda MS, Silva HS da. Participação em programas para a terceira idade: impacto sobre a cognição, humor e satisfação com a vida. Estud psicol (Campinas). 2010; 27(2): 207-214.

15. Schneider RH, Irigaray TQ. O envelhecimento na atualidade: Aspectos cronológicos, biológicos, psicológicos e sociais. Estud psicol (Campinas). 2008; 25(4): 585-593.

16. Gonçalves LHT, et al. Perfil da família cuidadora de idosos doente/fragilizado do contexto sociocultural de Florianópolis,SC. Texto Contexto Enferm. 2006; 15(4): 570-7.

17. Associação Brasileira de Estudos Populacionais (Brasil). Critério de Classificação Econômica Brasil, 2010.

18. Centers for Disease Control and Prevention. Behavioral Risk Factor Surveillance System Survey Questionnaire, 1999.

19. Matsudo S, et al. Questionário Internacional de Atividade Física (IPAQ): estudo de validade e reprodutibilidade no Brasil. Rev bras ativ fís saúde. 2001; 6(2): 5-18.

20. Almeida OP, Almeida SA. Short versions of the geriatric depression scale: a study of their validity for the diagnosis of a major depressive episode according to ICD-10 and DSM-IV. Int J Geriatr Psychiatry. 1999; 14(10): 858-6.

21. Bertolucci PHF, et al. O mini-exame do estado mental em uma população geral: impacto da escolaridade. Arq NeuroPsiquiatr. 1994; 52(1):1-7.

22. Strauss E, Sherman EMS, Spreen O. A Compendium of Neuropsychological Tests: Administration, Norms and Commentary (3rd ed.). New York: Oxford University Press, 2006.

23. Tombaugh TN. Trail Making Test A and B: normative data stratified by age and education. Arch Clin Neuropsychol. 2004;19(2):203-14.

24. Silva KCA, Lourenço RA. Tradução, adaptação e validação de construto do Teste do Relógio aplicado entre idosos no Brasil. Rev Saúde Pública. 2008; 42(5): 930-7.

25. Teixeira PC, et al. A pratica de exercícios físicos em pacientes com transtornos alimentares Rev psiquiatr clín. 2009; 34(4): 145-152.

26. Hillman $\mathrm{CH}$, et al. Physical activity and cognitive function in a cross-section of younger and older community-dwelling individuals. Health Psychol. 2006; 25(6): 678-87.

27. Machado JC, et al. Declínio cognitivo de idosos e sua associação com fatores epidemiológicos em Viçosa, Minas Gerais. Rev bras geriatr gerontol. 2011; 4(1): 109-121.

28. Leite, MT, et al. Qualidade de vida e nível cognitivo de pessoas idosas participantes de grupos de convivência. Rev bras geriatr gerontol. 2012; 15(3): 481-492.

29. Tombaugh TN. Trail Making Test A and B: normative data stratified by age andeducation. Arch Clin Neuropsychol. 2004; 19(2): 203-14.

30. Custodio EB, Malaquias Junior J, VOOS MC. Relação entre cognição (função executiva e percepção espacial) e equilíbrio de idosos de baixa escolaridade. Fisioter Pesqui. 2010; 17(1): 2010.

31. Ashendorf $L$, et al. Trail Making Test errors in normal aging, mild cognitive impairment, and dementia. Arch Clin Neuropsychol. 2008; 23(2): 129-37.

32. Valle EA, et al. Estudo de base populacional dos fatores associados ao desempenho no Mini Exame do Estado Mental entre idosos: Projeto Bambuí. Cad Saúde Pública. 2009; 25(4): 918-926.

33. Santos, IB dos. Oficinas de estimulação cognitiva em idosos analfabetos com transtorno cognitivo leve [dissertação]. Brasília: Universidade Católica de Brasília, 2010.

34. Diniz BSO, Volpe FM, Tavares AR. Nível educacional e idade no desempenho no Miniexame do Estado Mental em idosos residentes na comunidade. Rev psiquiatr Clín. 2007; 34(1): 13-17.

35. Lebrão ML, Duarte YAO. SABE - Saúde, Bem-estar e Envelhecimento - O Projeto Sabe no município de São Paulo: uma abordagem inicial. Brasília: Organização Pan-Americana da Saúde, 2003.

36. Leite VMM, et al. Depressão e envelhecimento: estudo nos participantes do Programa Universidade Aberta à Terceira Idade. Rev Bras Saude Mater Infant. 2006, 6(1): 31-38.

37. Carvalho FF, et al. Análise da percepção do estado de saúde dos idosos da região metropolitana de Belo Horizonte. Rev bras geriatr gerontol. 2012;15(2): 285-293.

38. Araújo NP de, et al. Aspectos sociodemográficos, de saúde e nível de satisfação de idosos institucionalizados no Distrito Federal. Rev Ciênc Méd 2008; 17(3-6): 123-132.

39. Oliveira, JGD de, et al. Correlação socioeconômica e antropométrica em idosos praticantes e não praticantes de exercícios físicos. Rev bras ativ fís saúde, 2013; 18(1): 121-131. 
40. Rosset, I, et al. Diferenciais socioeconômicos e de saúde entre duas comunidades de idosos longevos. Rev Saúde Pública. 2011; 45(2): 391-400.

41. Shibata A, et al. Prevalence and demographic correlates of meeting the physical activity recommendation among Japanese adults. J Phys Act Health 2009; 6(1): 24-32.

\section{Camila da Cruz Ramos de Araujo}

Endereço para correspondência - Rua: Evaldo Schaeffer, n²422, Ap 23, Bloco B10,

Bairro: Jardim Atlântico, CEP: 88095-350, Florianópolis, SC, Brasil.

E-mail: cami.ramosdearaujo@hotmail.com

Lattes: http://lattes.cnpq.br/0696770907183182

Camila da Silveira - milasilveiraa@hotmail.com

Joseani Paulini Neves Simas - joseanisimas@gmail.com

Alessandra Zappelini - azappelini@gmail.com

Silvia Rosane Parcias - sil_pa13@hotmail.com

Adriana Coutinho de Azevedo Guimarães - nanaguim@terra.com.br

Enviado em 28 de setembro de 2014.

Aceito em 29 de setembro de 2015. 
